\title{
Enfrentamento em crianças portuguesas hospitalizadas por câncer: comparação de dois instrumentos de avaliação
}

\author{
Coping in Portuguese children hospitalized \\ for cancer: A comparison of two \\ assessment instruments
}

\author{
Ana Sofia LIMA ${ }^{1}$ \\ Luísa BARROS 2 \\ Sônia Regina Fiorim ENUMO
}

\begin{abstract}
Resumo
Condições associadas à hospitalização e tratamentos de crianças com câncer têm sido identificadas como fontes de estresse, sendo pertinente estudar as estratégias de enfrentamento utilizadas por essas crianças. Este estudo objetivou comparar duas metodologias de avaliação do enfrentamento em contexto de hospitalização pediátrica. Dezenove crianças com câncer, com idade entre 6 e 12 anos, em tratamento em um hospital português, responderam ao Kidcope e ao Instrumento para Avaliação das Estratégias de Enfrentamento da Hospitalização. Além disso, também os cuidadores avaliaram a adaptação da criança ao hospital. Os resultados apontaram níveis reduzidos de perturbação comportamental e sofrimento, tanto na avaliação das crianças quanto na dos pais. A amostra apresentou diversidade de estratégias de enfrentamento, bem como preponderância de comportamentos facilitadores em ambas as escalas, com predominância de suporte social e distração, mas também a presença de pensamento mágico e ruminação. Ambas as escalas tiveram respostas semelhantes para suporte social e distração, mas não para outras estratégias. Embora não tenha ficado demonstrado que as escalas sejam equivalentes, ambas se mostraram úteis para fins clínicos.
\end{abstract}

Palavras-chave: Criança; Enfrentamento; Neoplasias; Pacientes hospitalizados.

\begin{abstract}
Conditions associated with hospitalization and treatment of children with cancer have been identified as sources of stress, thus the relevance of studying the coping strategies spontaneously used by these children. The present study aimed to compare two methods for evaluating coping in the context of pediatric hospitalization. A total of 19 children

$\nabla \nabla v$

1 Universidade de Lisboa, Faculdade de Psicologia, Programa de Pós-Graduação em Psicologia Clínica e da Saúde. Lisboa, Portugal.

2 Universidade de Lisboa, Faculdade de Psicologia, Programa de Pós-Graduação em Psicologia da Saúde. Alameda da Universidade, 1649-013, Lisboa, Portugal. Correspondência para/Correspondence to: L BARROS. E-mail: <lbarros@psicologia.ulisboa.pt>.

3 Pontifícia Universidade Católica de Campinas, Centro de Ciências da Vida, Programa de Pós-Graduação em Psicologia. Campinas, SP, Brasil.

Artigo elaborado a partir da dissertação de A.S. LIMA, intitulada "Avaliação de estratégias de coping em crianças com cancro: estudo exploratório". Universidade de Lisboa, 2009.
\end{abstract}


(aged 6 to 12) with cancer, attending a Portuguese hospital, answered the Kidcope and the Instrument for the Assessment of Coping Strategies during Hospitalization. Their caregivers evaluated how the children adapted to the hospital. Low levels of distress were observed in the evaluations of both caregivers and children. The sample showed various coping strategies and a number of facilitative behaviors in both scales, including social support and entertainment, but also wishful thinking and rumination. Both scales had similar responses in terms of social support and entertainment, but not for other strategies. Although the two scales were not found to be equivalents, both proved to be useful for clinical purposes.

Keywords: Child; Coping behaviors; Neoplasms; Hospitalized pacients.

As doenças oncológicas pediátricas implicam alterações importantes na vida da criança e da família. As condições associadas à hospitalização, tais como os tratamentos, o ambiente desconhecido, o contato com muitos profissionais diferentes, o afastamento de familiares e amigos e a ruptura das rotinas cotidianas associam-se a eventuais perdas e sofrimento. Essas experiências têm sido identificadas como fontes de stress para a criança e família (Barros, 2003; Motta \& Enumo, 2004, 2010; Phipps, 2007). Nesse contexto, o estudo das estratégias de enfrentamento (coping) utilizadas espontaneamente por crianças e adolescentes em situações de doença tem trazido uma contribuição importante para a intervenção nessa área (Wallandar \& Varni, 1992).

A maioria dos estudos sobre processos e estratégias de enfrentamento baseia-se na perspectiva teórica de Lazarus e colaboradores, que conceituam o enfrentamento em termos de suas funções primordiais: "esforços cognitivos e comportamentais, em constante mudança, para enfrentar as exigências específicas externas e/ou internas, que são avaliadas como desafiando ou excedendo os recursos da pessoa" (Lazarus \& Folkman, 1984, p.141). Esses esforços podem ser dirigidos para alterar a relação que o indivíduo estabelece com suas condições ambientais (enfrentamento focado no problema), ou para regular o sofrimento emocional, alterando o modo como o indivíduo interpreta a sua experiência, sem alterar a situação objetiva (enfrentamento focado na emoção).

No estudo do enfrentamento infantil, um enfoque desenvolvimentista considera que é necessário ter em conta que a infância e a adolescência são períodos do desenvolvimento caracterizados por mudanças importantes no nível cogni-

560 tivo, físico e psicossocial. Essas mudanças influen- ciam a forma como as crianças e os adolescentes vivenciam o stress e elaboram a avaliação subjetiva da situação e dos recursos disponíveis para enfrentá-la, assim como a escolha e utilização das estratégias (Aldwin, 2011; Barros, 2003).

A partir do modelo de avaliação cognitiva de Lazarus e Folkman (1984), outros modelos têm sido utilizados para orientar as pesquisas sobre o enfrentamento em crianças. Uma das perspectivas mais clássicas conceitua o comportamento do doente em termos de resposta de enfrentamento, numa dimensão que reflete a tendência do indivíduo para procurar ou evitar informação relevante para um evento estressante num continuum de busca-esquiva (Blount, Davis, Powers, \& Roberts, 1991; Suls \& Fletcher, 1985; Zimmer-Gembeck \& Skinner, 2011). Nessa linha, alguns estudos demonstram que crianças com câncer tendem a manifestar um aumento do estilo de enfrentamento evitante no início do tratamento (Aldridge \& Roesch, 2007; Worchel et al., 1988). Isto é, elas tendem a afastar sua atenção dos episódios negativos e indutores de estresse, assim como das reações psicológicas e somáticas a esses episódios. Para isso, recorrem essencialmente a estratégias de esquiva, como a distração e o pensamento mágico (Rodgers et al., 2012), mesmo que a informação evitada seja potencialmente relevante para a experiência durante os procedimentos médicos (Aldridge \& Roesch, 2007). Em contrapartida, os estudos com doentes que tiveram o diagnóstico de câncer há mais tempo e com sobreviventes demonstram um aumento das estratégias de aproximação e a associação desse aumento a uma melhor adaptação (Aldridge \& Roesch, 2007). Outros estudos mostram que as estratégias de suporte social são as mais eficazes dentre as usadas com frequência (Lindwall et al., 2012), mas também que as crianças usam frequentemente estratégias consideradas 
pouco eficazes (Bingen et al., 2012; Rodgers et al., 2012).

No geral, os resultados das pesquisas na área foram obtidos essencialmente em dois tipos de situação: relacionadas com tratamentos específicos, pontuais (transplante de medula, etc.) e com duração muito limitada, ou em estudos mais abrangentes sobre a adaptação, de médio e longo prazo, à doença oncológica e à sobrevivência. Foram encontrados apenas dois estudos sobre o coping durante o processo de hospitalização para tratamento do câncer (Motta \& Enumo, 2004; Motta, 2007).

Os estudos sobre o enfrentamento têm lidado com a complexidade desse conceito e a reduzida consistência entre a teoria, a pesquisa e a compreensão do fenômeno (Aldwin, 2011; Lazarus \& Folkman, 1984; Skinner, Edge, Altman, \& Sherwood, 2003). Atualmente, embora exista uma vasta produção científica sobre o tema, permanecem ainda por clarificar questões relacionadas com os instrumentos de medida, tais como a taxonomia inerente e a eficácia comparada (Folkman \& Moskowitz, 2004; Skinner et al., 2003). A maioria dos estudos é ateórico ou baseia-se apenas em teorias desenvolvidas para a população adulta (Barros, 2003; Compas, 2009). Também são utilizados questionários de autorrelato, entrevistas semiestruturadas, observação do comportamento e medidas complementares, como relatos de pais, professores e pares, que se diferenciam em termos conceituais e de abrangência ou foco, o que dificulta muito a comparação dos instrumentos (Skinner et al., 2003). Assim, o enfrentamento não pode ser considerado um comportamento específico, passível de observação, ou uma crença que pode ser relatada, pois é inerentemente multidimensional. Skinner et al. (2003) organizaram um modelo integrador a partir de uma rigorosa revisão da literatura e propuseram 13 categorias, apresentando definições claras, mutuamente exclusivas e de certa forma exaustivas: resolução do problema, suporte social, esquiva, distração, reestruturação cognitiva, ruminação, desamparo, afastamento social, regulação da emoção, busca por informação, negação, oposição e delegação. Com um enfoque desenvolvimentista, essa proposta é considerada "a mais compreensiva teoria do desenvolvimento das estratégias de enfrentamento na infância" (Aldwin, 2011, p.23).
Considerando a necessidade de aprofundar a potencialidade de medidas de enfrentamento infantil baseadas no autorrelato, este estudo pretendeu comparar duas metodologias de avaliação do enfrentamento em contexto de hospitalização pediátrica associada ao câncer. A primeira delas é a escala Kidcope (Spirito, Stark, \& Williams, 1988), um questionário de autorrelato estruturado, amplamente estudado e avaliado como abordagem bem estabelecida segundo a divisão da Pediatric Psychology da American Psychological Association (Blount et al., 2008). A outra metodologia, mais aberta e menos estudada, é o Instrumento para Avaliação do Enfrentamento da Hospitalização (AEH) (Motta \& Enumo, 2002, 2004, 2010), que recorre ao registro das verbalizações da criança para posterior análise de conteúdo. Pretendeu-se explorar as estratégias mais relatadas por esse grupo de crianças em tratamento do câncer, bem como comparar os resultados obtidos em cada instrumento e a adequação do seu uso com crianças em contexto hospitalar.

\section{Método}

Este estudo foi realizado no Serviço de Pediatria de um hospital público especializado em doenças oncológicas, localizado em Lisboa, Portugal. Atendendo aos objetivos, foi realizado um estudo exploratório, com recurso a uma amostra de conveniência, intencional e sequencial. O estudo foi aprovado pela Comissão de Ética do Hospital (Processo $n^{\circ} \mathrm{GIC} / 566$ aprovado em 7 de maio de 2009).

\section{Participantes}

A população deste estudo foi constituída pelas crianças em tratamento no Serviço de Pediatria do referido hospital, com idade entre 6 e 12 anos, de ambos os gêneros, e com vários tipos de câncer. O diagnóstico de câncer tinha sido estabelecido entre 2 e 72 meses antes ( $M=19$ meses), e todas as crianças estavam realizando tratamento quimioterápico. Apesar de não se pretender obter a representatividade da população atendida por esse 
serviço, definiram-se critérios de seleção intencional da amostra, de modo a incluir crianças com o diagnóstico de câncer: (a) em tratamento de quimioterapia entre 3-29 dias; (b) na faixa etária de 6-12 anos, de forma a ter capacidade de compreensão e linguagem suficientemente desenvolvidas para participar das tarefas propostas; (c) ter o consentimento informado dos pais e o assentimento da criança; (d) por questões éticas, apenas se abordaram as crianças que apresentavam alguma estabilidade emocional e comportamental. Foram considerados como critérios de exclusão das crianças: (a) estar na fase inicial de diagnóstico, dada a falta de experiência com a hospitalização; (b) estar em fase terminal da doença, para respeitar a integridade e eventual sofrimento dela e da família; (c) apresentar limitações impostas pela doença, impeditivas da realização das tarefas propostas; e (d) ter mais de 12 anos de idade, devido à inadequação dos instrumentos, que poderiam ser considerados como uma tarefa demasiado infantil para esses jovens.

Foram abordadas todas as 23 crianças que, no período do estudo, estavam em tratamento e cumpriam os critérios de inclusão. Dentre elas, 83\% completaram todas as tarefas, uma obteve realização incompleta e três recusaram-se a participar. Assim, a amostra final ficou constituída por 19 crianças, 10 do sexo feminino, todas de nacionalidade portuguesa, com idade entre 6-12 anos ( $M=10)$.

\section{Instrumentos}

O Kidcope (Spirito et al., 1988), é uma medida de autorrelato, que avalia as estratégias de enfrentamento utilizadas por crianças e adolescentes em várias situações. Baseia-se na conceituação de Lazarus e Folkman (1984), propondo-se como uma escala breve e de fácil aplicação, promovendo assim a complementaridade entre a pesquisa e a prática clínica. Foi utilizado em diversos estudos, incluindo duas populações clínicas pediátricas e majoritariamente adolescentes saudáveis $(n>330)$. Obteve índices de confiabilidade teste-reteste para intervalos pequenos ( 3 a 7 dias), com correlações entre

562 0,41 e 0,83; para intervalos maiores (>10 dias), cor- relações entre 0,15 e 0,43; além de correlações moderadas com outras medidas do enfrentamento infantil (Coping Strategies Inventory e Adolescent Coping Orientation for Problem Experiences Inventory, relativas à validade concorrente (Spirito et al., 1988). Não foram avaliados os índices de consistência interna, pois os itens do questionário abrangiam diferentes estratégias de enfrentamento (Spirito et al., 1988).

O Kidcope possui duas versões, uma para crianças de 7 a 12 anos, porém usada já a partir dos cinco anos (Pretzlik \& Sylva, 1999), e outra para adolescentes com idade superior a 12 anos. $O$ instrumento contém 15 itens, relacionados a 10 estratégias de enfrentamento: "esquiva" (distração, isolamento social, pensamento mágico e resignação), coping "negativo" (autocrítica e culpar os outros) e coping "ativo" (reestruturação cognitiva, resolução de problemas, regulação emocional e suporte social), abrangendo as estratégias de enfrentamento pediátrico mais frequentemente descritas na literatura.

Cada item tem duas escalas: (a) de frequência ("fizeste isto?"), com resposta "sim" (1) ou "não" (0), que fornece o número de estratégias de enfrentamento que a criança relata usar para lidar com a experiência de tratamento; e (b) de eficácia ("quanto ajudou?"), entre 0 ("nada") e 3 ("muito"), que indica o grau de eficácia autoavaliado das estratégias utilizadas.

Há três questões adicionais para medir o nível de adaptação ou de perturbação comportamental e sofrimento (distress) autoavaliado pela criança perante a situação problemática por ela descrita, relativas a: (a) ansiedade ("esta situação deixou-te nervoso?"); (b) tristeza ("esta situação deixou-te triste?"); e (c) raiva ("esta situação deixou-te irritado ou zangado?"). As respostas são cotadas em escala Likert de 1 ("nada") a 5 ("muitíssimo"), gerando uma pontuação total entre 3 e 15 pontos.

Não há normas disponíveis para o instrumento, pois sua aplicação se estende a diversas situações, com uso essencialmente de investigação (Spirito et al., 1988).

Esta aplicação inseriu-se num estudo mais amplo de sua adaptação e validação, ainda em 
curso, para a população de crianças e adolescentes portugueses, com diversas doenças crônicas, numa colaboração entre a Universidade de Coimbra e a Universidade de Lisboa (Carona, Silva, Barros, \& Canavarro, 2010). Ressalta-se que a validação desse instrumento é particularmente complexa, pois está sendo feita para uma cultura em que não existe outro instrumento, com objetivos similares, já validado, com que possa ser comparado - além do fato de o Kidcope não pressupor uma estrutura fatorial, nem ter uma consistência interna elevada.

\section{Avaliação das Estratégias de Enfrenta- mento na Hospitalização Pediátrica}

O "Instrumento para Avaliação das Estratégias de Enfrentamento na Hospitalização" foi elaborado por Motta e Enumo (2002), visando facilitar o acesso do psicólogo às experiências da criança hospitalizada, num período de tempo reduzido e de um modo agradável e motivador. Não sendo um instrumento psicométrico de avaliação direta de coping, visa fornecer informação indireta sobre os comportamentos mais frequentes durante a hospitalização, conforme relatados pela criança. A seleção desses comportamentos tem como pressuposto que alguns podem funcionar como estratégias facilitadoras e constituir um enfrentamento positivo, enquanto outros constituem estratégias potencialmente não facilitadoras ou de enfrentamento mais desadaptativo. Em aplicações com crianças brasileiras (6 a 12 anos), este instrumento - em formato impresso ou informatizado, ambos com versões para meninas e meninos -, revelou-se adequado para a identificação das emoções e comportamentos usualmente apresentados por crianças hospitalizadas com câncer e outras doenças crônicas, tendo sido utilizado em diferentes pesquisas (Carnier, 2010; Garioli, 2010; Moraes \& Enumo, 2008; Motta \& Enumo, 2002, 2010). Segundo Motta e Enumo (2004), sua proposta original baseiase igualmente na conceituação do enfrentamento de Lazarus e Folkman (1984) e na classificação de estratégias de enfrentamento proposta por Skinner et al. (2003).

O Instrumento para Avaliação das Estratégias de Enfrentamento na Hospitalização é constituído por um caderno com 20 pranchas $(18 \mathrm{~cm} \times 21 \mathrm{~cm})$, ilustradas com desenhos coloridos que representam possíveis comportamentos de uma criança durante a hospitalização. Dentre esses comportamentos, 10 são previamente classificados como facilitadores, na medida em que são potencialmente favoráveis ao enfrentamento da hospitalização: brincar, assistir TV, cantar e dançar, rezar, estudar, conversar, ouvir música, ler gibi, tomar remédio e buscar informações. São considerados não facilitadores aqueles comportamentos potencialmente desfavoráveis ao enfrentamento da hospitalização: chorar, sentir raiva, esconder-se, ficar triste, desanimar, fazer chantagem, pensar em fugir, sentir culpa, sentir medo e pensar em milagre.

Os estudos que pretenderam avaliar a validade de conteúdo do instrumento destacaram que o grupo de pranchas relativas aos comportamentos facilitadores apresenta uma validade superior (a representação gráfica é interpretada pelas crianças como estratégia de enfrentamento) à do grupo de pranchas representativas de comportamentos não facilitadores (Motta \& Enumo, 2004).

As respostas são obtidas em escala Likert, variando entre 0 ("nunca fiz, pensei ou senti") e 4 ("fiz, pensei ou senti sempre"). A criança deve responder à questão "quantas vezes fazes, pensas ou sentes o que estamos a ver no desenho quando estás aqui no hospital?" , colocando no caderno de respostas o número de círculos (de velcro, de tamanho e cores iguais) correspondentes ao ponto da escala selecionada: de 0 ("nenhuma") a 4 círculos ("sempre").

Para cada resposta, é igualmente solicitada à criança uma justificativa. O resultado total de comportamentos pode variar entre 0 e 80 , sendo até 40 para os comportamentos facilitadores e até 40 para os não facilitadores. A análise qualitativa das justificativas fornecidas para cada prancha permite identificar as estratégias de enfrentamento entre as 13 categorias propostas por Skinner et al. (2003): solução do problema, busca de suporte, esquiva, distração, reestruturação cognitiva, ruminação, desamparo, isolamento social, regulação da emoção, busca de informação, negociação, oposição e dele- 
gação. Após, processa-se o cálculo da frequência de participantes que produziram verbalizações integradas em cada categoria.

Foi ainda utilizada uma escala subjetiva de um item para avaliar a percepção do cuidador principal sobre a adaptação da criança à hospitalização, solicitando-lhe que a comparasse à adaptação das outras crianças. A resposta era dada numa escala Likert, com 5 pontos, variando de 1 ("muito pior que as outras crianças") até 5 ("muito melhor que as outras crianças").

\section{Procedimentos}

As crianças foram abordadas sequencialmente, numa sala de convívio onde brincavam enquanto esperavam o tratamento ou outros procedimentos, estando acompanhadas por um familiar que preencheu o protocolo de consentimento informado. Em seguida, obteve-se o assentimento da criança, após os esclarecimentos sobre a pesquisa. Os dados de identificação da criança e a caracterização de sua condição clínica foram obtidos junto do adulto e complementados com informações clínicas. Foi ainda solicitado ao adulto que respondesse à escala subjetiva sobre a adaptação da criança ao hospital e aos tratamentos.

A pesquisadora conduzia a criança para uma sala reservada e iniciava com ela a conversa, começando por questionar se conhecia o motivo da hospitalização. Em seguida, foi aplicado o AEH, individualmente. Cada aplicação durou cerca de 30 minutos, sendo gravada em áudio para facilitar a análise das justificativas verbais da criança, que foram posteriormente transcritas para a folha de registo. Ao final, foi aplicado o Kidcope, no formato de entrevista, para ajudar a criança a compreender o conteúdo dos itens, com duração aproximada de 10 minutos. Foi-lhe solicitado que se recordasse da última experiência de tratamento realizado no hospital e que mantivesse essa recordação presente enquanto respondia às questões.

Os dados de caráter quantitativo foram analisados com recurso ao Statistical Package for the Social Sciences (SPSS) versão 17.0. Inicialmente, foi

564 feita uma análise descritiva dos resultados obtidos nos dois instrumentos e na resposta do cuidador sobre a adaptação da criança. Posteriormente, investigou-se a existência de associações estatisticamente significativas entre duas variáveis, através do Coeficiente de correlação $r$ de Pearson para variáveis de intervalo e o Teste não paramétrico do Qui-quadrado de Pearson para variáveis nominais. Quando as condições de aplicabilidade do Qui-quadrado falharam, utilizou-se o Teste Exato de Fisher, procurando assim melhorar a análise estatística feita a partir do Qui-quadrado de Pearson. Para investigar a existência de diferenças estatisticamente significativas entre médias da mesma amostra mediante duas condições diferentes, utilizou-se o Teste $t$ de Student para amostras emparelhadas. Para investigar a existência de diferenças estatisticamente significativas entre médias de uma variável quantitativa numa variável dicotômica, ou seja, em grupos diferentes de participantes (definidos e agrupados por idade, por regime de tratamento e por tempo de diagnóstico), utilizou-se o Teste $t$ de Student para amostras independentes (Pestana \& Gageiro, 2008). Foram verificadas as condições para realizar os vários testes, e valores de $p$ inferiores a 0,05 foram considerados como significativos.

Para a análise dos resultados do AEH utilizou-se uma combinação de abordagem quantitativa, já referida, e de abordagem qualitativa. Os dados de caráter qualitativo, ou seja, as justificativas das crianças às respostas fornecidas em cada uma das pranchas foram transcritas e incluídas nas categorias de enfrentamento propostas por Skinner et al. (2003), conforme Tabela 1. Posteriormente, processou-se o cálculo da frequência de participantes que produziram verbalizações integradas em cada categoria de enfrentamento.

\section{Resultados}

Avaliação parental da adaptação da criança: globalmente, os pais consideraram a adaptação do filho tão boa ou melhor que a das outras crianças (variação = 3-5; $M=3,53$; mediana = 3,00; $\mathrm{DP}=0,772$ ).

Análise dos resultados do Kidcope: nas três escalas do questionário Kidcope, a média de per- 
Tabela 1

Análise qualitativa das justificativas no AEH: categorias de enfrentamento, verbalizações das crianças e frequência de cada categoria $(N=19)$

\begin{tabular}{ll}
\hline Categorias & Definição \\
\hline 1. Distração (DIS) & $\begin{array}{l}\text { Envolvimento em atividades alternativas prazerosas } \\
\text { e possíveis no ambiente hospitalar, como forma de } \\
\text { tentar lidar com a situação estressante. }\end{array}$ \\
$\begin{array}{ll}\text { 2. Busca de Suporte } & \text { Ações da criança em direção ao alvo de suporte; } \\
\text { (BSU) } & \text { relatos que demonstram a aceitação do suporte } \\
& \text { social disponível (procura de ajuda instrumental, } \\
& \text { conselhos, conforto e contato com as pessoas). } \\
\text { 3. Ruminação (RUM) } & \text { Foco passivo e repetitivo nos aspectos negativos da } \\
& \text { situação, com ênfase nos danos e perdas (exposi- } \\
& \text { ção a procedimentos médicos aversivos, afastamen- } \\
\text { to dos familiares, quimioterapia etc.). } & \text { Açães dirigidas para a resolução do problema: com- } \\
\text { portamentos de adesão ao tratamento, ação dirigida } & \text { à minimização da situação estressante, quando a } \\
\text { Problemas (RPR) } & \text { criança relata que não se esconde dos médicos, e } \\
& \text { indicação do foco no tratamento e na cura da do- } \\
\text { ença. }\end{array}$
\end{tabular}

5. Busca de Informação Tentativas de aprender mais sobre a situação (BIN) estressante, quer por meio de perguntas diretas, quer por meio de observação dos acontecimentos.

6. Esquiva (ESQ) Não envolvimento com a situação estressante; "fuga cognitiva", negação e ações diretas de evitamento.

7. Reestruturação Tentativas ativas da criança mudar sua percepção Cognitiva (RCO) sobre a situação estressante, no sentido de vê-la de maneira mais positiva.

8. Isolamento Social (ISO)

Ações dirigidas a manter-se distante das outras pessoas ou de impedi-las de saber sobre a situação estressante ou seus efeitos emocionais.

9. Regulação da Emoção (REM)

Esforços da criança para influenciar seu sofrimento emocional e expressar suas emoções construtivamente, no momento e lugar apropriados, fazendoa sentir-se melhor.

10. Desamparo (DES) Passividade, confusão, interferência ou exaustão cognitiva, desânimo, pessimismo.

11. Oposição (OPO)

Comportamentos de projeção, agressão, reação de raiva, descarga e atribuição de culpa às outras pessoas.

12. Delegação (DEL) Comportamentos de dependência, procura de ajuda mal adaptativa, reclamações, queixas e autopiedade.

13. Negociação (NEG) Tentativas ativas de fazer um acordo entre suas necessidades e as restrições impostas pelo contexto da hospitalização.

\begin{tabular}{ll} 
Exemplos de verbalizações & $f$ \\
\hline "Porque não gosto de não fazer nada e de estar a & 19
\end{tabular}
pensar que tenho de estar no hospital e fico nervosa, por isso brinco".

"...Também é bom falar com os amigos, ... ao menos, assim fico mais calma, sinto-me mais à vontade".

"Porque a quimioterapia dá-me... ahh... fico nauseada e depois não me apetece comer nada e depois tenho dor e a comida não é lá muito do meu agrado".

"Agora tenho pensado em enfrentar a doença!".

"Porque quero saber o que é a minha doença e foi o que eu fiz sempre que a mãe acabava de falar com o médico...".

"Não penso muito neles, porque os pensamentos podem não ser verdade".

"Porque não tenho de pensar negativo, tenho de brincar e esquecer o máximo possivel isto, tenho de pensar na positiva, não posso nunca chorar por uma coisa que já está a passar".

"Porque eu brinco sempre sozinha. (Q) Aqui não conheço ninguém".

"Sinto-me um bocado triste cada vez que vou para o hospital de dia e chega a altura de ser picada, fico um pouco nervosa; mas, de resto, quando estou aqui no Lions, estou a brincar e esqueço-me que estou no hospital e entretenho-me com os meninos".

"Tenho pensamentos de não me curar muito rápido e tenho medo de não me curar... e... são os únicos pensamentos que tenho...".

"Sempre que acordo das picadas que são para adormecer, fico assim e nervosa, começo a chamar nomes aos enfermeiros; mas é só quando levo estas picadas".

"Se os meninos ou as meninas quiserem brincar comigo, eu brinco para não ficarem zangados comigo, nem tristes".

Chantagem: "Porque me apetece... (Q) comida".

x


turbação identificada pelas crianças apresentou um valor relativamente baixo (variação = 3-13; $M=5,63$; $\mathrm{DP}=3,32$.

Esses resultados mostram o uso predominante de estratégias de enfrentamento avaliadas pelas crianças como bastante ou moderadamente eficazes, em especial as estratégias de enfrentamento ativo (suporte social, reestruturação cognitiva e regulação emocional) e de esquiva (distração e pensamento mágico). Mostram, ainda, o uso reduzido de estratégias que as crianças avaliaram como pouco eficazes, de enfrentamento negativo (autocrítica) (Tabela 2).

Para verificar possíveis relações entre os resultados obtidos na escala de distress e as estratégias de enfrentamento, compararam-se os dados das duas escalas do Kidcope, organizados segundo as duas dimensões de enfrentamento propostas pelos autores: estratégias de esquiva e de enfrentamento ativo. Não foi possível realizar a análise para o enfrentamento negativo porque apenas três partici- pantes relataram utilizar esse tipo de estratégia. Resultados elevados na escala de perturbação associaram-se ao relato elevado de estratégias de esquiva $(r=0,487 ; p<0,05)$.

\section{Análise dos resultados do AEH}

No Instrumento para Avaliação das Estratégias de Enfrentamento na Hospitalização, observou-se um valor relativamente baixo para o total de comportamentos relatados pelas crianças $(M=28,16)$, sendo médio para os comportamentos facilitadores da hospitalização $(M=18,68)$ e baixo para os comportamentos não facilitadores $(M=9,47)$ (Tabela 3 ). Constatou-se predominância significativa de comportamentos facilitadores $(t=5,156 ; p<0,05)$, sendo que apenas duas crianças referiram mais comportamentos não facilitadores do que facilitadores. Os comportamentos mais referidos remetem a estratégias de enfrentamento, como a distração (brincar, conversar e ver TV) e a resolução de problemas (tomar remédio); e os menos referidos remetem a es-

Tabela 2

Estratégias de enfrentamento e sua autoeficácia segundo crianças com câncer, no Kidcope ( $N=19)$

\begin{tabular}{|c|c|c|c|c|c|c|c|}
\hline \multirow{2}{*}{$\begin{array}{l}\text { Tipo de } \\
\text { enfrentamento }\end{array}$} & \multirow{2}{*}{$\begin{array}{l}\text { Estratégias } \\
\text { de enfrentamento }\end{array}$} & \multicolumn{2}{|c|}{ Estratégias identificadas } & \multicolumn{4}{|c|}{ Eficácia autoatribuída das estratégias } \\
\hline & & $f(0-1)$ & $\mathrm{Fa}$ & Nada (0) & Pouco (1) & Muito (2) & $\mathrm{Md}$ \\
\hline \multirow[t]{4}{*}{ Esquiva } & Distração & 18 & 0,95 & 3 & 3 & 13 & 2 \\
\hline & Pensamento mágico & 18 & 0,95 & 4 & 5 & 10 & 2 \\
\hline & Isolamento social & 9 & 0,47 & 13 & 4 & 2 & 0 \\
\hline & Resignação & 6 & 0,32 & 14 & 4 & 1 & 0 \\
\hline \multirow[t]{2}{*}{ Negativo } & Autocrítica & 3 & 0,16 & 19 & 0 & 0 & 0 \\
\hline & Culpar os outros & 0 & 0 & 19 & 0 & 0 & 0 \\
\hline \multirow[t]{4}{*}{ Ativo } & Suporte social & 19 & 1 & 0 & 3 & 16 & 2 \\
\hline & Reestruturação cognitiva & 16 & 0,84 & 4 & 5 & 10 & 2 \\
\hline & Regulação emocional & 16 & 0,84 & 5 & 6 & 8 & 1 \\
\hline & Resolução de problemas & 13 & 0,68 & 7 & 6 & 6 & 1 \\
\hline
\end{tabular}

Tabela 3

Frequência média de respostas das crianças às pranchas do $\mathrm{AEH}(N=19)$

\begin{tabular}{lccccc}
\hline Tipos de comportamentos de coping & $N$ & Mínimo & Máximo & M & DP \\
\hline Facilitadores (0-40) & 19 & 7 & 30 & $18,68^{*}$ & 6,725 \\
Não Facilitadores (0-40) & 19 & 2 & 25 & $9,47^{*}$ & 5,511 \\
Total de comportamentos (0-80) & 19 & 14 & 42 & 28,16 & 9,518 \\
\hline
\end{tabular}

Nota: * $p<0,05$; Teste $t$ de Student.

566 AEH: Instrumento para Avaliação do Enfrentamento da Hospitalização; M: Média; DP: Desvio-Padrão. 
tratégias de enfrentamento por esquiva (esconder-se, pensar em fugir e pensar em milagres) e distração (canto e dança).

A identificação das estratégias de enfrentamento das crianças, feita com base nas suas justificativas, mostrou, para cada criança, uma média de 6,89 estratégias de enfrentamento. As estratégias mais relatadas foram: busca de suporte, distração, ruminação e resolução de problemas; e as menos relatadas foram negociação, delegação e oposição. Houve, assim, preponderância de estratégias associadas a comportamentos facilitadores; a ruminação foi o único comportamento não facilitador com frequência elevada (Tabela 1).

Um objetivo deste estudo foi averiguar até que ponto as respostas a cada um dos instrumentos seriam semelhantes. Considerando que, em ambos os instrumentos, há coincidência na designação de seis estratégias (resolução de problemas, suporte social, distração, reestruturação cognitiva, isolamento social e regulação emocional), comparou-se a fre-quência de crianças que reportaram utilizar cada uma dessas estratégias simultaneamente em ambos os instrumentos e, posteriormente, comparou-se a frequência daquelas que as reportaram em apenas um dos instrumentos. Para o total das estratégias não houve associação significativa entre respostas semelhantes nos dois instrumentos, embora se tenha verificado concordância absoluta nas respostas para o suporte social e concordância muito elevada para a estratégia de distração.

\section{Discussão}

Este trabalho explorou a utilização de dois instrumentos ainda não estudados na população portuguesa -, Kidcope et al., 1988) e o AEH (Motta \& Enumo, 2002, 2004, 2010). Eles permitiram avaliar o nível de perturbação e as estratégias de enfrentamento utilizadas pelas crianças com câncer em tratamento hospitalar, numa amostra de 19 crianças, com idade entre 6 e 12 anos.

A quase totalidade das crianças demonstrou conhecer o motivo da hospitalização/tratamento, referindo ser o tratamento, a doença ou ainda am- bos, em proporção inferior. A maioria das crianças relatou níveis de perturbação reduzidos, corroborando assim diversos estudos sobre a adaptação psicológica de crianças com câncer (Aldridge \& Roesch, 2007; Phipps, 2007; Phipps, Farclough, \& Mulhern, 1995). Todos os pais consideraram a adaptação do filho ao hospital tão boa ou melhor que a de outras crianças, dado este consistente com os reduzidos níveis de perturbação relatados pelas crianças. Adicionalmente, as crianças reportaram níveis intermédios de eficácia das estratégias utilizadas e preponderância de estratégias que avaliaram como mais eficazes (Kidcope) ou de comportamentos classificados teoricamente como facilitadores da adaptação ao hospital (AEH). Assim sendo, a percepção dos cuidadores sobre a adaptação dessas crianças ao hospital parece ser congruente com a avaliação que elas fizeram de si mesmas. Os resultados confirmaram os obtidos por Motta e Enumo (2010) com o AEH, para uma população de crianças hospitalizadas para tratamento de câncer.

No presente estudo, as crianças com níveis de distress mais elevados reportaram usar mais estratégias de esquiva, o que contraria outros estudos que demonstram que a esquiva está associada a níveis reduzidos de perturbação em crianças com câncer (Aldridge \& Roesch, 2007; Phipps, 2007; Phipps et al., 1995). Não se verificou associação entre o enfrentamento ativo e os níveis de perturbação. A maioria das crianças reportou uma diversidade de estratégias, englobando não só as de esquiva, como também de enfrentamento ativo e, em menor número, de enfrentamento negativo. Tais resultados são consistentes com os obtidos por Mota e Enumo (2010). No entanto, o fato de a maioria das crianças reportar níveis baixos de perturbação e diversidade de estratégias limita a interpretação desses resultados de associação.

Verificou-se o relato preponderante de estratégias centradas na emoção ou de controle secundário - distração, procura de suporte, pensamento mágico, reestruturação cognitiva e regulação emocional. Bingen et al. (2012) também destacaram maior uso de distração e pensamento mágico em crianças submetidas a transplante de células-tronco hematopoiéticas. No mesmo sentido, Aldridge e 
Roesch (2007) constataram que, no grupo de crianças com diagnóstico relativamente recente de câncer, como é o caso desta amostra, estas usavam mais estratégias centradas na emoção e de esquiva - em detrimento daquelas centradas no problema, presentes unicamente sob a forma de resolução de problemas. As estratégias centradas na emoção permitem alterar a forma como a criança reage e interpreta o problema, alterando o significado da sua experiência, sem alterar a situação objetiva (Lazarus, 1966). É nesse sentido que maioria dos autores sugere que elas são mais eficazes quando existem aspectos da situação estressante que não são modificáveis, tal como acontece com o câncer e a consequente realização de procedimentos médicos dolorosos (Band \& Weisz, 1988; Compas, 2009; Harbeck-Weber, Fisher, \& Dittner 2003).

De modo geral, as crianças recorrem mais a estratégias que também avaliam como eficazes. Essa associação é mais evidente nas estratégias de suporte social, distração, reestruturação cognitiva, pensamento mágico e regulação emocional. Bingem et al. (2012) também observaram que a estratégia de pensamento mágico era das mais usadas, embora fosse considerada das menos eficazes.

Comparando os dois instrumentos e o modo de sua aplicação, constatou-se que o Kidcope, por ser uma escala breve e de fácil manejo, permite examinar uma grande variedade de estratégias de enfrentamento que cada criança poderá utilizar ou não, de forma rápida e objetiva. Além disso, esse instrumento permite avaliar a eficácia subjetiva atribuída a cada estratégia. Por se fundamentar num modelo teórico bem definido, o Kidcope permite interpretar de forma clara os resultados e compará-los com outras escalas de enfrentamento baseadas no modelo de Lazarus e Folkman (1984). No entanto, apesar de Pretzlik e Sylva (1999) considerarem que a versão para crianças pode ser aplicada a partir dos cinco anos, as crianças mais novas desta amostra - com idade de seis anos -, revelaram alguma dificuldade na compreensão dos itens.

Por sua vez, o AEH consiste numa metodologia lúdica, apelativa e inovadora, eventualmente mais interessante para crianças mais novas, 568 e tem a grande vantagem de permitir obter dados qualitativos subjetivos sobre a experiência idiossincrática de cada criança. No entanto, seus resultados podem ser um pouco mais difíceis de interpretar, pelo fato de esse instrumento se basear num conceito de enfrentamento mais amplo, que considera não somente as respostas, comportamentos ou instâncias de enfrentamento relatadas, mas também pretende deduzir as estratégias de enfrentamento funcionalmente relacionadas e hierarquicamente superiores (Skinner et al., 2003). Em alguns casos, a relação entre as ações representadas nas pranchas (ficar triste, com raiva, com medo, desanimar ou chorar) e a estratégia que lhes é atribuída pelo modelo conceitual (neste caso, a ruminação) não é direta, pois a definição da estratégia de enfrentamento é deduzida pelo aplicador a partir da justificativa dada pela criança. Algumas dessas pranchas podem representar reações emocionais que as crianças identificam porque as experienciaram, sem contudo tê-las utilizado como estratégia de enfrentamento - isto é, não são ações intencionais para se sentirem melhor ou se adaptarem ao hospital ou ao tratamento.

Tal como foi descrito anteriormente, ambos os instrumentos incluem seis estratégias de enfrentamento que os respectivos autores designam do mesmo modo e que deveriam, assim, avaliar as mesmas estratégias. Contudo, constatou-se que as crianças deram respostas semelhantes apenas para duas: suporte social e distração.

Esses resultados podem estar relacionados, por um lado, com o fato de as crianças não terem compreendido totalmente os itens do Kidcope e/ou os desenhos do AEH, ou de lhes terem atribuído significados diferentes. Mas, também, podem implicar alguma fragilidade na validade de conteúdo desses itens.

A análise dos resultados obtidos com o AEH evidencia um padrão de respostas semelhante ao obtido pelas autoras do instrumento num estudo com crianças com câncer (Motta \& Enumo, 2004). Contudo, três pranchas obtiveram pontuações muito mais baixas que no estudo brasileiro: esconder-se, pensar em fugir, e cantar e dançar - talvez pelo fato de essas crianças não utilizarem esses comportamentos e pensamentos para lidar com o tratamento e/ou a hospitalização. 
Dado o pequeno tamanho da amostra, não se pode concluir desde já que existam diferenças culturais significativas, embora essa hipótese deva ser explorada em outros estudos. Com efeito, os resultados também poderão ser explicados pelo fato de as crianças portuguesas interpretarem os desenhos de forma diferente das crianças brasileiras em especial o desenho da criança que se esconde do médico, que foi frequentemente interpretado como uma brincadeira, e não como uma ação de esquiva.

Relativamente ao cantar e dançar, a maioria das crianças relatou que, apesar de ser esta uma brincadeira divertida e uma boa estratégia de distração, não é aplicável ao contexto hospitalar, como também observado no estudo de Motta e Enumo (2004).

Este estudo atingiu o objetivo proposto de explorar os níveis de perturbação e as estratégias mais relatadas por um grupo de crianças em tratamento do câncer, através do recurso a dois instrumentos de avaliação. Foram encontrados níveis reduzidos de perturbação, tanto na avaliação da criança pelos pais, como naquela efetuada pela própria criança. Em estudos anteriores, foi demonstrado que esses níveis baixos estavam associados ao aumento do estilo de enfrentamento de esquiva e suporte social, o que também se observou nesta amostra. Embora as crianças com níveis mais elevados de stress relatassem maior uso de estratégias de esquiva, o delineamento do estudo não permitiu identificar a direção dessa associação nem estabelecer alguma relação de causalidade. Verificou-se que as crianças utilizavam uma grande diversidade de estratégias, incluindo não só aquelas de esquiva, como também de enfrentamento ativo e, em menor número, negativo. As estratégias centradas na emoção foram as mais relatadas pelas crianças. Atendendo a que vários aspectos do tratamento e do contexto hospitalar não são modificáveis ou controláveis, a escolha desse tipo de estratégia parece estar associada aos bons níveis de adaptação da amostra. No entanto, a adaptação da criança à hospitalização e ao tratamento resulta da combinação de diferentes variáveis individuais e contextuais, sendo as estratégias de enfrentamento apenas um desses múltiplos determinantes (Aldridge \& Roesch, 2007).

No que diz respeito à comparação entre os dois instrumentos de avaliação do coping na situação de hospitalização e tratamento, esse objetivo foi apenas parcialmente atingido. Embora ambos os instrumentos avaliem o mesmo conceito e embora seus resultados se aproximem, não ficou demonstrado que eles sejam equivalentes.

Mais uma vez, evidenciou-se a ideia defendida por Lazarus e Folkman (1984) e Skinner et al. (2003) sobre a falta de consenso na literatura acerca do significado do enfrentamento, o que se reflete na reduzida consistência entre teoria, pesquisa e compreensão do fenômeno, dificultando a tarefa de tornar mais homogênea a investigação e, consequentemente, de comparar resultados de diferentes estudos, o que contribuiria para o progresso na área. Nesta pesquisa, tais questões ficaram evidentes tanto na análise da construção e estrutura dos dois instrumentos, quanto na discussão dos resultados obtidos.

Por outro lado, importa referir que ambos os instrumentos podem ser considerados como metodologias adequadas à comunicação e compreensão da criança em tratamento médico, por promoverem a expressão de suas experiências subjetivas de uma forma não abusiva, que salvaguarda sua integridade e bem-estar. É nesse sentido que ambos se revelam instrumentos de avaliação úteis, quer para efeitos de investigação, quer para fins clínicos. Enquanto o AEH parece oferecer maiores potencialidades para o uso clínico e a exploração individual de diversos tipos de estratégias, o Kidcope, pela sua fundamentação teórica sólida e rapidez de aplicação, pode ser mais útil para a pesquisa e a caracterização.

Esses resultados devem ser analisados com precaução, atendendo às características deste estudo exploratório. Anota-se que sua principal limitação prende-se à reduzida dimensão da amostra e a seu caráter de conveniência. Outra limitação relaciona-se com o fato de que, por questões éticas, a amostra do estudo foi apenas constituída por crianças que apresentavam alguma estabilidade emo- 
cional e comportamental, o que pode ter enviesado a pesquisa e explicar os valores elevados de adaptação. Finalmente, atendendo a que se trata de um estudo transversal e que a avaliação da eficácia de cada estratégia é apenas feita pela criança, é difícil compará-lo com outros estudos em que as estratégias de enfrentamento foram usadas como preditores de adaptação posterior e em que foram usados indicadores objetivos dessa adaptação.

Acredita-se que seja importante dar continuidade a esta linha de estudo, aumentando o tamanho da amostra de modo a poder controlar variáveis clínicas e tempo de tratamento, bem como aprofundando o estudo das diferenças entre crianças de idades diferentes. Essa direção permitirá compreender se ambos os instrumentos são igualmente adequados para crianças de idades diferentes, ou se as diferenças na estrutura e forma de resposta de cada instrumento justificam que algum deles seja mais adequado para determinada faixa etária.

\section{Referências}

Aldridge, A. A., \& Roesch, S. C. (2007). Coping and adjustment in children with cancer: A meta-analytic study. Journal of Behavioral Medicine, 30(2), 115-129.

Band, E. B., \& Weisz, J. R. (1988). How to feel better when it feels bad: Children's perspectives on coping with everyday stress. Development Psychology, 24(2), 247-253.

Aldwin, C. (2011). Stress and coping across de life span. In S. Folkman (Ed.), The Oxford handbook of stress, health, and coping (pp.15-34). New York: The Oxford University Press.

Barros, L. (2003). Psicologia pediátrica: perspectiva desenvolvimentista. Lisboa: Climepsi.

Bingen, K., Kent, M., Rodday, A. M., Ratichek, S., Kupst, M. J., \& Parson, S. (2012). Children's coping with hematopoietic stem cell transplant stressors: Results from the journeys to recovery study. Children's Health Care, 41(2), 145-161.

Blount, R. L., Davis, N., Powers, S. W., \& Roberts, M. C. (1991). The influence of environmental factors and coping style on children's coping and distress. Clinical Psychology Review, 11, 93-116.

Blount, R. L., Simons, L. E., Devine, K. A., Janiste, T., Cohen, L. L., Chambers, C. T., \& Hayutin, L. G. (2008). Evidence-based assessment of coping and stress in Pediatric Psychology. Journal of Pediatric Psychology, 570 33(9), 1021-1045.
Carnier, L. E. (2010). Stress e coping em crianças hospitalizadas em situação pré-cirúrgica e stress do acompanhante: estabelecendo relações (Dissertação de mestrado não-publicada). Universidade Estadual Paulista Júlio de Mesquita Filho, Bauru. Recuperado em fevereiro 25, 2011, de http://www2.fc.unesp.br/ BibliotecaVirtual/ArquivosPDF/DIS_MEST/DIS MEST20100723_CARNIER\%20LUCIANA\%20ESGALHA.pdf

Carona, C., Silva, N., Barros, L., \& Canavarro, C. (2010). Kidcope: questionário de avaliação do coping em crianças e adolescentes (Documento não-publicado). Instituto de Psicologia Cognitiva, Desenvolvimento Vocacional e Social, Universidade de Coimbra.

Compas, B. E. (2009). Coping, regulation, and development during childhood and adolescence. In E. A. Skinner, \& M. J. Zimmer-Gembeck (Eds.), Coping and the development of regulation. New Directions for Child and Adolescent Development, 124, 87-99. San Francisco: Jossey-Bass. Retrieved January 25, 2011, from http://www.interscience.wiley.com

Folkman, S., \& Moskowitz, J. T. (2004). Coping: Pitfalls and promise. Annual Reviews Psychology, 55, 745-774.

Garioli, D. (2010). O impacto da dor nas funções executivas e sua relação com as estratégias de enfrentamento em crianças com anemia falciforme (Dissertação de mestrado não-publicada). Universidade Federal do Espírito Santo, Vitória.

Harbeck-Weber, C., Fisher, J. L., \& Dittner, C. A. (2003). Promoting coping and enhancing adaptation to illness. In M. C. Roberts (Ed.), Handbook of Pediatric Psychology (pp.99-118). New York: The Guilford Press.

Lazarus, R. S. (1966). Psychological stress and the coping process. New York: McGraw-Hill.

Lazarus, R. S., \& Folkman, S. (1984). Stress, appraisal and coping. New York: Springer.

Lindwall, J., Russell, H., Kelly, E., Klaas, S., Mulcahey, M. J., Betz, R., \& Vogel, L. (2012). Coping and participation in youth spinal cord injury. Topics in Spinal Cord Injury Rehabilitation, 18(3), 220-231.

Moraes, E. O., \& Enumo, S. R. F. (2008). Estratégias de enfrentamento da hospitalização em crianças avaliadas por instrumento informatizado. Psico USF, 13(2), 221-231.

Motta, A. B., \& Enumo, S. R. F. (2002). Brincar no hospital: Avaliação do enfrentamento da hospitalização. Psicologia: Saúde e Doenças, 3(1), 23-41.

Motta, A. B., \& Enumo, S. R. F. (2004). Câncer infantil: uma proposta de avaliação das estratégias de enfrentamento da hospitalização. Estudos de Psicologia (Campinas), 21(3), 193-202.

Motta, A. B., \& Enumo, S. R. F. (2010). Intervenção psicológica lúdica para o enfrentamento da hospitalização em crianças com câncer. Psicologia: Teoria e Pesquisa, 26(3), 445-454.

Pestana, M., \& Gageiro, J. (2008). Análise dos dados para Ciências Sociais: A complementaridade do SPSS. Lisboa: Edições Silaba. 
Phipps, S. (2007). Adaptative style in children with cancer: Implications for a Positive Psychology Approach. Journal of Pediatric Psychology, 32(9), 1055-1066.

Phipps, S., Farclough, D., \& Mulhern R. K. (1995). Avoidant coping in children with cancer. Journal of Pediatric Psychology, 20(2), 217-232.

Pretzlik, U., \& Sylva, K. (1999). Paediatric patients distress and coping during medical treatment: A self report measure. Archives of Disease in Childhood, 81, 525-527.

Rodgers, C., Norville, R., Taylor, O., Poon, C., Hesselgrave, J., Gregurich, M. A., \& Hockenberry, M. (2012). Children's coping strategies for chemotherapy-induced nausea and vomiting. Oncology Nursing Forum, 39(2), 202-209.

Skinner, E., Edge, K., Altman, J., \& Sherwood, H. (2003). Searching for the structure of coping: A review and critique of category systems for classifying ways of coping. Psychological Bulletin, 129, 216-269.

Spirito, A., Stark, L. J., \& Williams, C. (1988). Development of a brief coping checklist for use with pediatric populations. Journal of Pediatric Psychology, 13(4), 555-574.
Suls, J., \& Fletcher, B. (1985). The relative efficacy of avoidant and non-avoidant coping strategies: A meta-analysis. Health Psychology, 4, 249-288.

Wallander, J. L., \& Varni, J. W. (1992). Adjustment in children with chronic physical disorders: Programmatic research on a disability-stress-coping model. In A. M. LaGreca, L. Siegal, J. L. Wallander, \& C. E. Walker (Eds.), Stress and coping in child health (pp.279-298). New York: Guilford Press.

Worchel, F. F., Nolan, B. F., Wilson, V. L., Purser, J., Copeland, D. R., \& Pfefferbaum, B. (1988). Assessment of depression in children with cancer. Journal of Pediatric Psychology, 13, 101-112.

Zimmer-Gembeck, M. J., \& Skinner, E. A. (2011). The development of coping across childhood and adolescence: An integrative review and critique of research. International Journal of Behavioral Development, 35, 1-17.

Recebido em: 4/3/2013

Versão final em: 10/7/2013

Aprovado em: 20/8/2013 
